\title{
Design and Construction of Vehicle Loading Monitoring System Using Load Sensor and GSM
}

\author{
Ebenezer Narh Odonkor ${ }^{1}$, \\ Department of Electrical and Electronics Engineering \\ Takoradi Technical University, Takoradi \\ Ghana \\ Willie K. Ofosu \\ Penn State Wilkes-Barre 1269 Old 115 Dallas, \\ PA
}

\begin{abstract}
Vehicle overloading is one of the major problems confronting many developing nations and Ghana is not an exception. Two attendant problems are accidents, and damage to road infrastructure. Weighing stations may be the answer to these conditions, but few are used for measuring the weight of some specific vehicles in Ghana. A vehicle loading monitoring detection using a load sensor and GSM model with an automatic engine lock system is proposed in this study as a solution. The proposed system will detect vehicle overloading and automatically call the owner of the vehicle or the road safety officer in that event. Proteus software is used for designing and simulating the circuit while programming is done using the Arduino software. Microcontroller ATMEGA 328 serves as the brain of the design and Global System for Mobile Communications (GSM) module serves as the communication link. A Liquid Crystal Display (LCD) is used to display the status of the vehicle. In addition, a buzzer is to sound an alarm when vehicle overloading is detected. The system has facilities to lock a vehicle engine automatically.
\end{abstract}

Keywords: Keypad, GSM, Load Sensor, LCD and Microcontroller

\section{Introduction}

The most commonly known causes of road traffic accidents in Ghana include overloading of vehicles, gross indiscipline by drivers on the roads, drunk driving, over speeding, talking on a cell phone while driving, wrong parking, and the breakdown of vehicles in the middle of roads (Dzokpo, 2014). The transportation of goods and passengers by road has increased over the years due to rapid development. As the need for road transport grows, vehicle overloading has increased. Overloaded vehicles reduce the lifespan of road infrastructure and pose a threat to road users (Zhong, Huo, \& Deng, 2013). According to statistics, $70 \%$ of traffic accidents are caused by vehicle overloading, making it one of the key issues of traffic management (Huang, 2016). Overloading is only checked at static weighing stations, which are sometimes situated far away from the loading points of vehicles (Huang, 2019). Due to this fact, truckers and trucking companies (both commercial and private) must be held to the highest safety standards. It is common for those in the trucking industry in Ghana to attempt to subvert safety standards (Annan, 2017). Any vehicle that is overloaded, for example, an 18-wheeler vehicle which is too heavy, cannot stop easily when necessary, and is more likely to skid off the road during a minor swerve to avoid potholes. Even though there are laws in place which dictate weight and height limits, these laws may be circumvented in the interest of getting a load delivered quickly. This paper focuses on a vehicle loading monitoring system for commercial and private vehicles in Ghana using a load sensor and GSM technology.

\section{Background of the Study}

The current rate of growth in the logistics industry and the increment in the world's population mean that a lot more vehicles need to be used (Goh \& Ling, 2003). Therefore, vehicle overloading is also becoming more serious. Consequently, the rapid deterioration of roads in Ghana can be attributed to vehicle overloading (Zhong, Huo \& Deng, 2013). The increment in traffic accidents which are caused by vehicle overloading has become one of the major issues for traffic management in Ghana. According to Huang (2019), a traditional static weighing system is used in weighing vehicle(s). Huang (2019) posits that there are many unsolved problems in existing weighing technologies where measuring instruments have to be installed in the concrete of roads to measure the weight of vehicles. This makes them inconvenient to maintenance. Weighing stations are not easily accessible in Ghana for heavy duty vehicles (Ghana Highway Authority, 2018).

To compound this issue, these stations are also purposely designed for only long axle vehicles (Huang, 2019). 
As a result, this study proposes a vehicle loading monitoring system using a load sensor (Infrared IR sensor) with an automatic engine lock system and GSM model for call alert to monitor their weight when being loaded. This system will help vehicle owners and road safety personnel to detect a vehicle's status when overloading detection occurs through a call alert notification. Furthermore, an early warning of overloading will be issued to the vehicle's driver or the person loading the vehicle to avoid exceeding the vehicle's gross weight.

\section{Related Work}

Siquan, Min and Chuundong (2017) discussed the geophone sensor which was a new type of vehicle overloading detection system that had the ability to detect overloaded vehicles and spontaneously transfer the relevant data to a server or screen. However, it does not alert the vehicle owner from a distance. Ramya, Palaniappan and Karthick (2012) examined a system that provides vehicle cabin safety based on an embedded system that monitors the level of toxic gases such as carbon monoxide (CO), liquefied petroleum gas (LPG) and alcohol within vehicles. Their system has the ability to send alert information through an alarm system during dangerous situations, and also send an SMS to an authorized person through the GSM technology. Ioan, Cioc and Visan (2006) examined a low-cost automotive localization system using GPS and GSM-SMS services. Their system permits localization of the automobile and transmits its position to the owner on his or her mobile phone as a short message (SMS). Vikhankar and Chaudhari (2016) conducted research on Advanced Vehicle Security System with Theft Control and Accident Notification. In their study, accident notification was done through GPS and GSM technologies to prevent increasing theft of vehicles at public places.

\section{Block Diagram}

Figure1 below shows the block diagram of the blue print of the proposed vehicle overloading monitoring system with an automatic engine lock and a call alert. It consists of a microcontroller, GSM module, power supply, keypad, LCD display, DC motor (car engine), load sensor, buzzer and an engine control block.

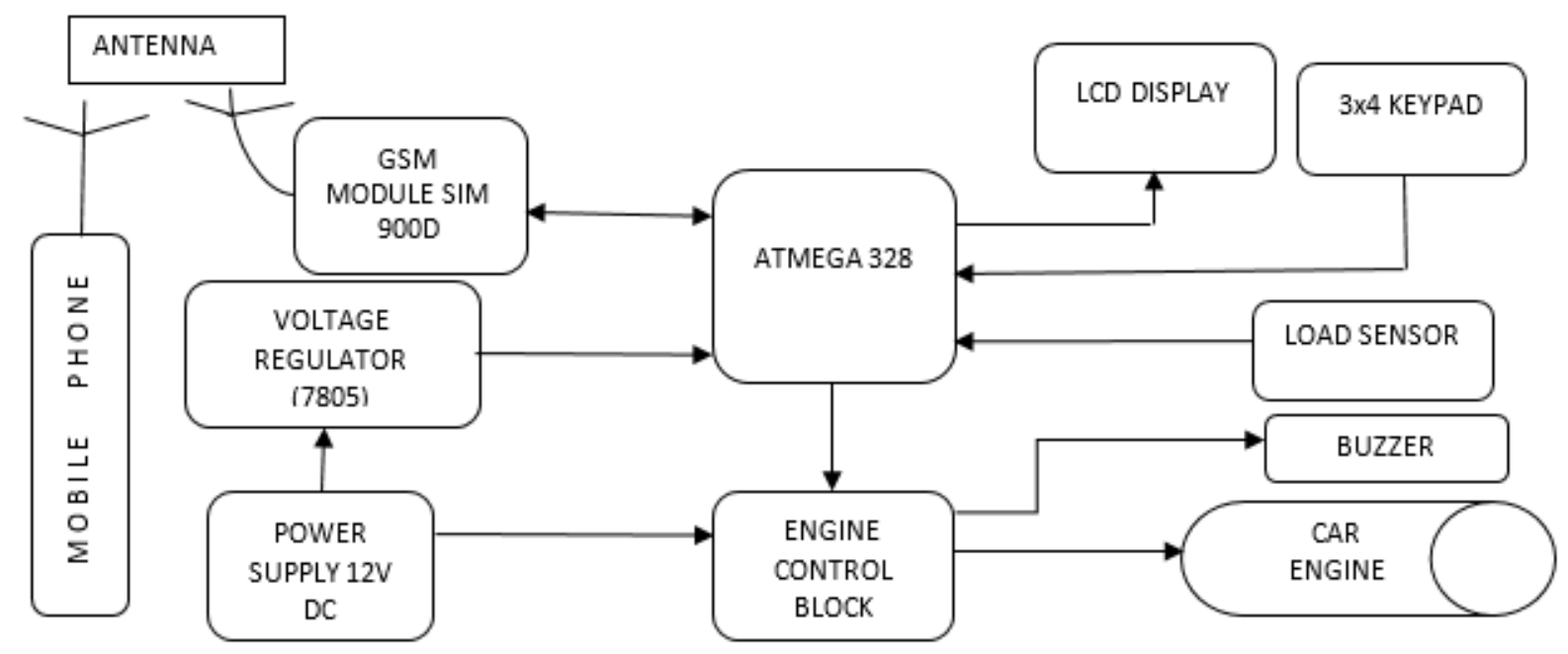

Figure 1: The Block Diagram of the Vehicle Load Monitoring System with an automatic Engine Lock System based on GSM

\section{Description of Block Diagram}

The power supply consists of a 12volt D.C battery which supplies the entire circuit, and the engine starter. The ignition key is used to open and close the circuit for current to flow from the battery. The supply is fed into a voltage regulator (7805) through pin VI. The voltage is regulated from 12VDC to 5VDC and then sent to the microcontroller ATMEGA 328 , which is programmed to receive an input from the load sensor. Again, this supply voltage is connected to the car engine and the buzzer through the engine control relay. The load sensor is used to detect or sense the change in the weight of the vehicle. If the voltage signal from the sensor is more than the reference voltage, the sensor sends a signal to the microcontroller. The microcontroller receives a signal from the sensor and then provides an output with respect to the input signal given by the sensor. The microcontroller then sends this signal to display the status of the vehicle on the liquid crystal display (LCD). When overloading is detected, the microcontroller sends a signal to the engine control block which consists of engine start relay which provides latching for the engine when started.

The engine switch relay turns on the vehicle engine (motor) under normal conditions. When overloading is detected, the engine is turned off, and the buzzer turned on respectively. The engine recovery relay is used to unlock the vehicle engine when overloading is detected. The reset code is set to recover the system to its normal operation state using keypad 
characters. The engine lock relay is energized to break the engine circuit from receiving power supply when the ignition key is turned on under overloading conditions. The GSM module SIM 900D receives the signal from the microcontroller to launch a call to the vehicle owner's mobile phone. The authorized person (owner of the vehicle) then uses the keypad to reset the engine recovery password to the microcontroller to send a signal to deactivate the engine recovery relay to bring the vehicle engine to normal operation mode and deactivate the buzzer as well.

\section{Circuit Design}

Figure 2 shows the circuit diagram of the Vehicle Loading Monitoring with an automatic engine lock system based on GSM.

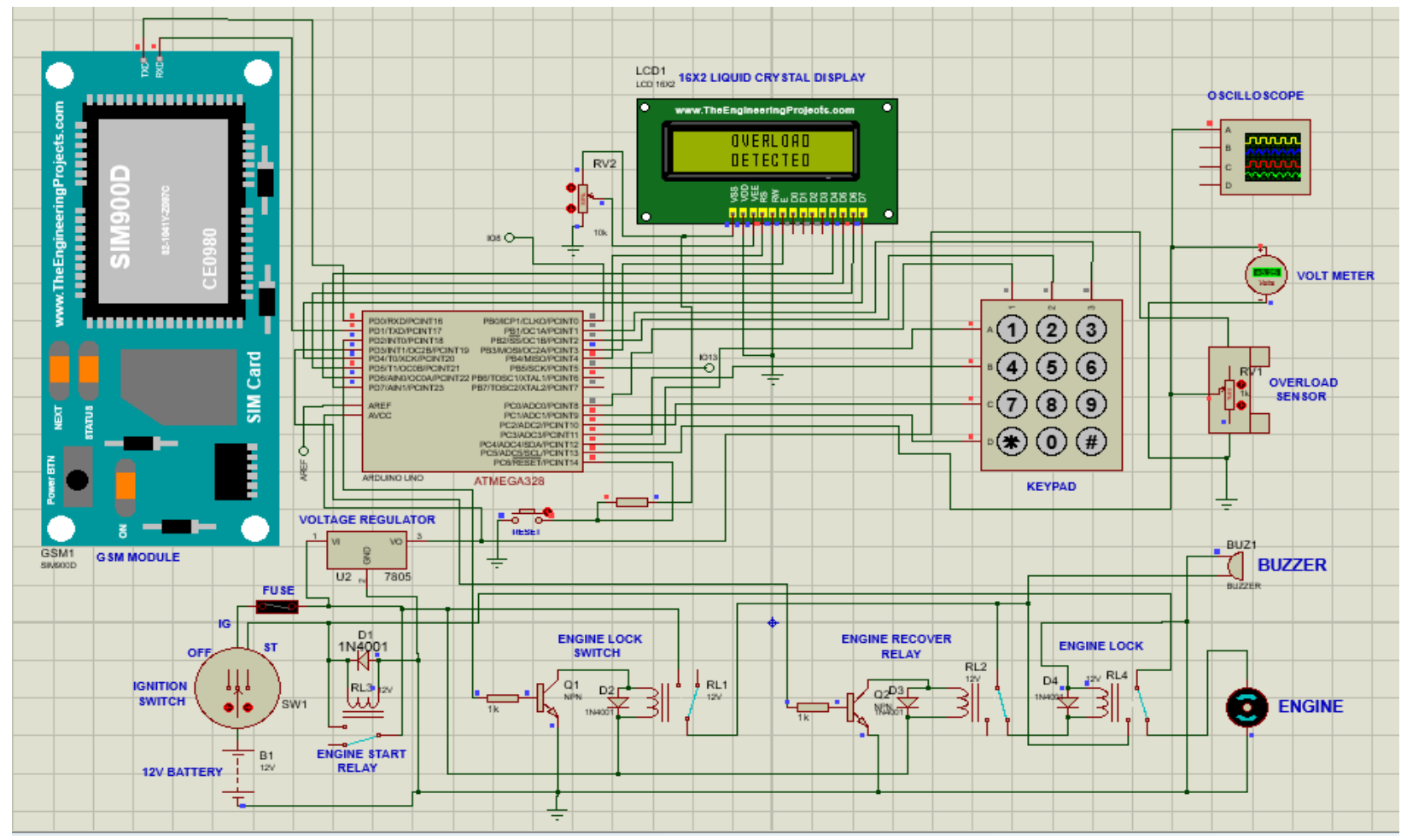

Figure 2: Circuit diagram of Vehicle Loading Monitoring with an automatic engine lock system based on GSM

The power supply is taken from the 12VDC battery that supplies the engine start relay. This relay provides a seal-in for current to flow through the normally closed (NC) contact of the engine lock relay (RL4) to the car engine. On ignition, supply voltage from the battery goes to terminal (1) of the voltage regulator (7805) while terminal 3 is connected to the AVCC terminal of the ATMEGA328 microcontroller and terminal 2 connected to ground. The 7805 reduces the input voltage of $12 \mathrm{VDC}$ to 5VDC to supply the microcontroller ATMEGA328. At normal condition when overloading is not detected, the signals (voltage) from the load sensor to the ATMEGA328 sensor input pin INT13 will be within the set point so the output signals from the microcontroller to the Liquid Crystal Display (LCD) shows the normal condition of the vehicle. When overloading is detected, a signal (voltage) which is above the set point will be sent to the microcontroller from the overloading sensor. The output signal from pin INT18 of the microcontroller is sent to the engine lock switch relay (RL1) through the base resistor to the NPN transistor Q1 to switch it on to energize the relay coil. When the coil of the relay is energized, its normally opened contact closes and power at the normally opened contact of the RL1 is transferred to the buzzer to sound an alarm. At this point, the coil of the engine lock is energized and that opens the engine circuitry to prevent power from reaching the engine, as a result establishing the engine lock condition. When this happens, the Liquid Crystal Display (LCD) indicates overloading detected. The microcontroller sends a signal to the GSM module SIM900D connected to the transmitter terminal INT16 and the receiver terminal INT17 to automatically call the owner of the vehicle (an authorized person). To bring the engine back to its normal operating mode, the authorized person must enter the recovery code using the keypad. When overloading is detected, before the recovery code is entered, the load in the vehicle must be reduced (or removed) so that the code will be able to activate the system. When the correct code is dialed, it sends a signal from INT19 terminal of the ATMEGA328 microcontroller to the base of the transistor Q2 through the $1 \mathrm{k} \Omega$ resistor to switch it on. When the transistor Q2 is switched on, current flows through the collector of Q2 to the engine recovery relay coil to energize it. The normally closed (NC) contact of Q2 opens to prevent current from flowing through the engine lock relay which de-energizes the engine lock relay. This closes the 
vehicle engine circuitry to allow the engine to turn on when the ignition key is activated. The reset button is used to reset the ATMEGA328 microcontroller when an error occurs. Variable resistor (RV2) is used to increase and decrease the resolution (brightness) of the LCD screen. The DC motor and the buzzer are supplied with 12VDC from the battery anytime the ignition key is activated. A voltmeter is connected to measure the voltage signal of the load sensor anytime a load is placed in the vehicle.

\section{Results}

Table 1 below shows the experimental results of the overloading sensor. The load sensor reading is presented in percentage (\%) against the rising level of the sensor.

Table 1: Load sensor readings are in percentages (\%) against the rising level of the sensor (weight of load).

\begin{tabular}{llll}
\hline $\begin{array}{l}\text { NUMBER OF } \\
\text { EXPERIMENT }\end{array}$ & $\begin{array}{l}\text { SENSOR (IR) READINGS } \\
\text { IN PENCENTAGE (\%) }\end{array}$ & WEIGHT OF LOAD (g) & $\begin{array}{l}\text { OVERLOAD } \\
\text { DETECTION } \\
\text { STATE }\end{array}$ \\
\hline 1 & & & Not detected \\
2 & 0 & 0 & Not detected \\
3 & 10 & 103 & Not detected \\
4 & 20 & 206 & Not detected \\
5 & 30 & 309 & Not detected \\
6 & 40 & 412 & Detected \\
7 & 50 & 515 & Detected \\
\hline
\end{tabular}

The graphical representation of the experimental results shown in Table 1 is shown in Figure 3 below.

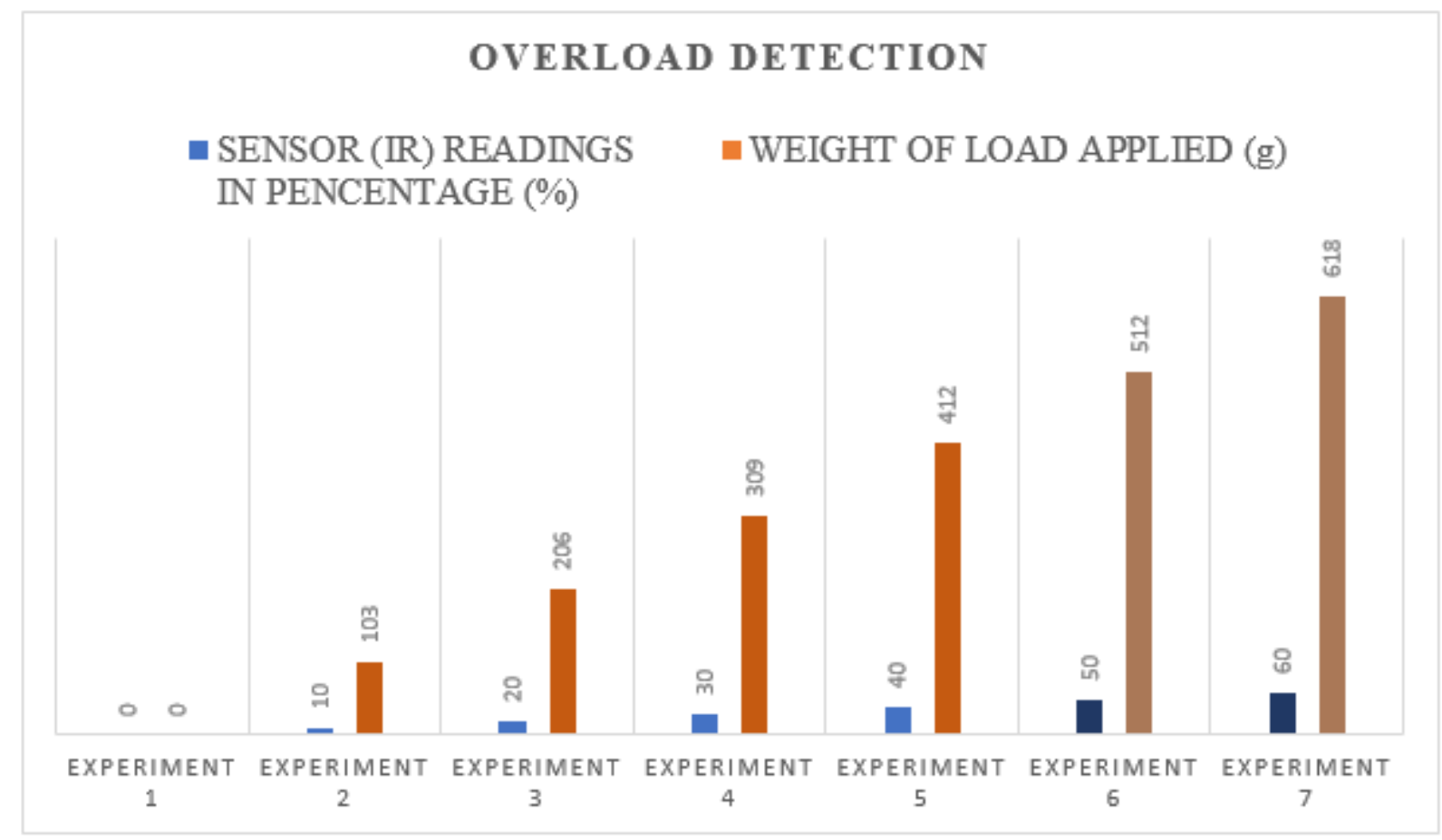

Figure 3: Graph of load sensor reading in percentage (\%) against rising level of sensor.

The values tabulated in Table 2 below are the results obtained from the experiment conducted on the sensitivity of the load sensor readings in percentage against voltage when the vehicle (Prototype) is loaded with different sizes and weight of loads in grams $(\mathrm{g})$. 
Table 2: Experimental results of the sensitivity of the load sensor

\begin{tabular}{llll}
\hline $\begin{array}{l}\text { NUMBER OF } \\
\text { EXPERIMENT }\end{array}$ & $\begin{array}{l}\text { SENSOR (IR) READINGS } \\
\text { IN PENCENTAGE }(\%)\end{array}$ & $\begin{array}{l}\text { SENSING SIGNAL IN } \\
\text { VOLTA (V) }\end{array}$ & $\begin{array}{l}\text { OVERLOAD } \\
\text { DETECTION } \\
\text { STATE }\end{array}$ \\
\hline 1 & 0 & 0.0 & Not detected \\
2 & 10 & 0.5 & Not detected \\
3 & 20 & 1.0 & Not detected \\
4 & 30 & 1.5 & Not detected \\
5 & 40 & 2.0 & Not detected \\
6 & 50 & 2.5 & Detected \\
7 & 60 & 3.0 & Detected \\
\hline
\end{tabular}

The graphical representation of the experimental results of the sensitivity of the load sensor tabulated in Table 2 is as shown in Figure 4 below.

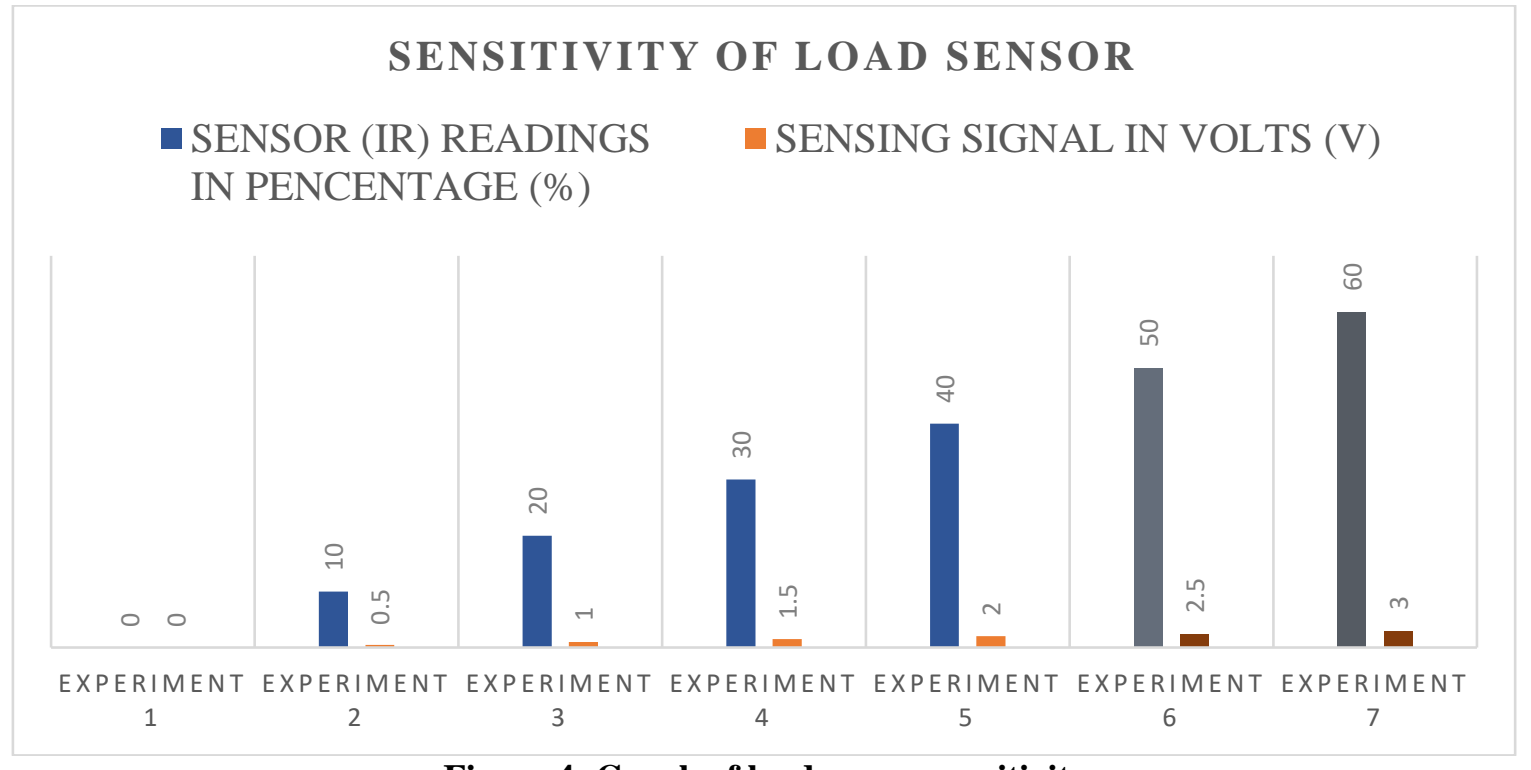

Figure 4: Graph of load sensor sensitivity

Figure 5 below shows the final packaging of the prototype of the vehicle loading monitoring system using load sensor and GSM with an automatic engine lock.

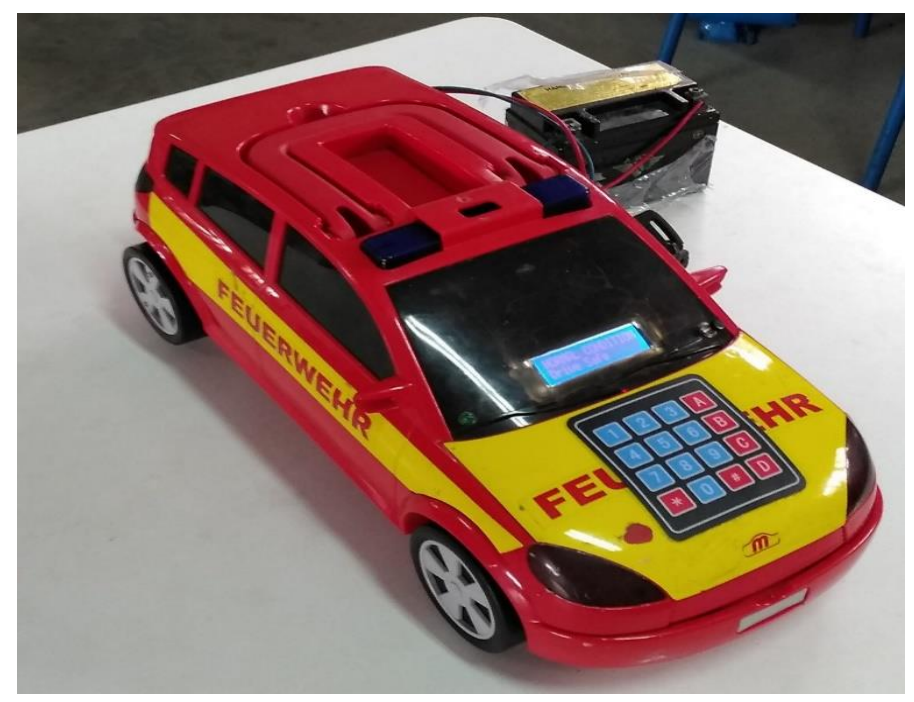

Figure 5: Prototype of vehicle load monitoring with an automatic engine lock system based on GSM 


\section{Discussion}

Table 1 show the result for a prototype vehicle overloading prevention system that uses a load sensor with an automatic engine lock and call alert system. In each of the tests, the load sensor was subjected to different weights until overloading was detected. At each percentage, the status of the vehicle was sent from the virtual terminals of the microcontroller ATMEGA 328 to the screen of the Liquid Crystal Display (LCD) to indicate the condition of the vehicle until overloading was detected. In all, seven experiments were conducted at different ranges of the load sensor levels. The $0 \%$ gives a zero gram $(0 \mathrm{~g})$ of weight indicating the normal load sensor condition of the vehicle when there is no load in the vehicle. The LCD indicates that overloading is not detected. The percentage up to $49 \%$ which gives its equivalent weight of $498 \mathrm{~g}$ which shows the maximum weight of load the vehicle can carry for which overloading is not detected. The microcontroller compares the set point value to the signal from the sensor. The microcontroller is programmed such that if the difference in value exceeds the normal weight of the vehicle, it sends information about the excess weight to the engine lock relay to activate it as it is programmed to lock the engine of the vehicle. Any percentage with its corresponding weight above $50 \%$ and $515 \mathrm{~g}$ will indicate the maximum level at which overloading is detected. The system will then call the owner of the vehicle automatically. Again, Figure 3 shows the minimum and maximum levels within which overloading can be detected. Table 2 shows the results for the sensitivity of the load sensor. The above sensor readings in percentage against sensing signal readings in volts $(\mathrm{V})$ shows that, when the sensor was varied from $0 \%$ to $50 \%$, there was a constant increment of 0.5 volts. When the sensor percentage was $50 \%$ and above, a constant $0.5 \mathrm{~V}$ was again recorded. This indicates good sensitivity as the sensitivity of a system or circuit is the smallest unit of a given parameter that can be meaningfully detected by an instrument when used. Hence, the load sensor used in this project work can detect 0.5 volt change in the input voltage. Figure 4 shows the variation of the sensitivity of the load sensor. The time delay for the load sensor to respond to overloading was due to the integrated circuit (IC) of the microcontroller used in this study. The above results are clear indications that the logical flow (flow chart) model of vehicle overloading monitoring system with GSM and a call alert system using load sensor (IR sensor) with an automatic engine lock system has been achieved. The experimental results were analyzed using Microsoft Excel.

Comparing the results obtained in Tables 1 and 2, it is obvious that when any vehicle used in Ghana has a load sensor fixed in it, there will be no need for a vehicle driver to drive some distance before weighing the load carried by his or her vehicle. This would help to ease the stress imposed on vehicles and road infrastructure. When all vehicles in Ghana are fixed with load sensors the lifespan of both vehicles and roads will be increased.

\section{Conclusion}

The testing and validation of the logical (flow chart) model was successfully achieved by using a simulation software package (Proteus) and Arduino programming software. The experimental device after it was designed; it was simulated before used in construction of the artifact. Design and construction of the vehicle loading monitoring system was successfully accomplished. The prototype worked exactly as a real-time system during demonstration. GSM SIM900D module was used as communication link for the system to call the vehicle owner automatically under overload condition. The vehicle load monitoring system is proposed with the aim of solving the problem of the inability of some vehicle drivers to access axle weighing stations to weigh the load their vehicles carry due to the limited number of weighing stations available in Ghana. To help stop vehicle overloading in Ghana, it is recommended, the proposed vehicle loading monitoring system is implemented. The engine lock system could prevent vehicle breakdown as well as accidents. This could potentially replace existing technology where drivers have to wait for hours at weighing stations before their vehicles' gross weight is captured using weighing sensors installed in roadway concrete. Implementing this technology in vehicles in Ghana or anywhere in the world will help to reduce overloading, which usually leads to road accidents and infrastructure deterioration.

\section{References}

Dzokpo, I. K. (2014). Overloading of Heavy goods vehicles deteriorating roads in Ghana. Retrieved fromhttps://www.newsghana.com.gh/overloading-heavy-goods-vehicles-deteriorating-roads-ghana. Assessed on $10-9-2018$

Zhong H J, Huo Y and Deng C C 2013. Computer Measurement \& Control 4. p.75-77. Retrieved from https://iopscience.iop.org/article/10.1088/1742-6596/887/1/012021/pdf. Accessed on 10/12/2019

Huang, H., Zhou, J., Zhang, J., Wangxi, X., Zhixing, C., \& Ningning, L. (2019). Effects of Revised Toll-by-Weight Policy on Truck Overloading Behavior and Bridge Infrastructure Damage Using Weigh-in-Motion Data: A Comparative Study in China. Advances in Civil Engineering. Volume 2019, Article ID 5910463, 13 pages

Retrieved from: https://doi.org/10.1155/2019/5910463. Accessed on 6/4/2019 
James Annan 2017; Road Accidents- A Major Public Safety Concern. Retrieved from https://www.modernghana.com/newsphoto/1/50/NzcxMzQx. last accessed on 13/10/19

Ghana Highway Authority (2018). axle load. Retrieved from

http://www.highwys.gov.gh/index.php.

Siquan, H., Kong, M., \& Chundong, S. (2017). Design of vehicle overload detection system based on geophone. IOP Conf. Series: Journal of Physics: Conf. Series 887 (2017) 012021 DOI :10.1088/1742-6596/887/1/012021

V.Ramya, B. Palaniappan, K. Karthick. "Embedded Controller for Vehicle In-Front Obstacle Detection and Cabin Safety Alert System", International Journal of Computer Science \& Information Technology (IJCSIT) Vol 4, No 2, April 2012. Accessed on 15/1/2019

Soichi Kubota, Yoshiharu Okamoto, Hideo Oda of National Institute of Information and Communication Technology (NICT), Japan, "Safety Driving Support System Using REID for Prevention of Pedestrian-involved Accidents", 6th International Conference on ITS Telecommunications Proceedings 2006.

IoanLita, Ion BogdanCioc, Daniel Alexandru Visan, "A New Approach of Automobile Localization System Using GPS and GSM/GPRS Transmission," vol. 8, pp. 415-422, 2006.

A. D. Vikhankar1, Prof. R. P. Chaudhari (2016) 'Advanced Vehicle Security System with Theft Control and Accident Notification', International Journal of Innovative Research in Science, Engineering and Technology (An ISO 3297: 2007 Certified Organization) Vol. 5.

M. Goh and C.Ling (2003), "Logistics development in China", International Journal of Physical Distribution \& Logistics Management, Vol. 33 No. 10, pp. 886-917. 